\title{
VOLUME ESTIMATE ABOUT SHRINKERS
}

\author{
XU CHENG AND DETANG ZHOU \\ (Communicated by Chuu-Lian Terng)
}

\begin{abstract}
We derive a precise estimate on the volume growth of the level set of a potential function on a complete noncompact Riemannian manifold. As applications, we obtain the volume growth rate of a complete noncompact selfshrinker and a gradient shrinking Ricci soliton. We also prove the equivalence of weighted volume finiteness, polynomial volume growth and properness of an immersed self-shrinker in Euclidean space.
\end{abstract}

\section{INTRODUCTION}

In this paper, we estimate the volume of complete noncompact Riemannian manifolds without assuming curvature conditions. Let $(M, g)$ be an $n$-dimensional complete noncompact Riemannian manifold on which there exists a nonnegative smooth function $f$ satisfying "shrinking soliton" conditions.

Let $\Delta_{f}$ be the linear operator defined by

$$
\Delta_{f} u=\Delta u-\langle\nabla f, \nabla u\rangle .
$$

It is known that the operator $\Delta_{f}$ is symmetric with respect to the weighted measure $e^{-f} d v$ on the space of smooth functions with compact support. Denote the lower level set of $2 \sqrt{f}$, its volume and its weighted volume by

and

$$
\begin{aligned}
& D_{r}=\{x \in M: 2 \sqrt{f}<r\}, \\
& V(r)=\operatorname{Vol}\left(D_{r}\right)=\int_{D_{r}} d v,
\end{aligned}
$$

$$
V_{f}(r)=\operatorname{Vol}_{f}\left(D_{r}\right)=\int_{D_{r}} e^{-f} d v .
$$

We get the volume growth estimate of the set $D_{r}$ and the finiteness of the weighted volume of $M$ in the following.

Theorem 1.1 (Theorem 2.1). Let $(M, g)$ be a complete noncompact Riemannain manifold. If $f$ is a proper $C^{\infty}$ function on $M$ satisfying $|\nabla f|^{2} \leq f$ on $D_{r}$ for all $r$ and

$$
\Delta_{f} f+f \leq k
$$

for some constant $k$, then $M$ has finite weighted volume; that is,

$$
V_{f}(M)=\int_{M} e^{-f} d v<+\infty
$$

Received by the editors July 4, 2011.

2010 Mathematics Subject Classification. Primary 53C20.

Both authors were partially supported by CNPq and FAPERJ, Brazil. 
and

$$
V(r) \leq C r^{2 k}
$$

for $r \geq 1$, where $C$ is a constant depending only on $\int_{M} e^{-f} d v$.

The classical volume comparison theorems in Riemannian geometry are one of the basic ingredients of the analysis on manifolds. It is usually done by assuming that $M$ is an $n$-dimensional Riemannian manifold with Ricci curvature being bounded below. In our theorem, instead of assuming curvature conditions, we assume the existence of some functions which arise naturally in the study of gradient shrinking Ricci solitons of Ricci flow and self-shrinkers of mean curvature flow. Both gradient shrinking Ricci solitons and self-shrinkers are important in the singularity analysis of Ricci flow and mean curvature flow respectively ([10, [11, [12]) and recently have been studied very much (see [6], 7], survey papers [1, 2], etc.). We observe that both of them satisfy inequality (1), and so Theorem 1.1 can be applied to them. Besides, Theorem 1.1 is of independent interest. We only need to find a proper function on $M$ satisfying the assumption of the theorem.

As the first application, we consider self-shrinkers for mean curvature flow (MCF) in $\mathbb{R}^{n+1}$. Recall that a one-parameter family of hypersurfaces $\Sigma_{t} \subset \mathbb{R}^{n+1}$ flows by mean curvature if

$$
\partial_{t} x=-H \mathbf{n},
$$

where $\mathbf{n}$ is the outward unit normal and $H$ is the mean curvature. The flow $\Sigma_{t}$ is said to be a self-shrinker if

$$
\Sigma_{t}=\sqrt{-t} \Sigma_{-1}
$$

for all $t<0$. This is equivalent to $\Sigma=\Sigma_{-1}$ satisfying the equation

$$
H=\frac{\langle x, \mathbf{n}\rangle}{2} \text {. }
$$

We also call the hypersurface $\Sigma$ a self-shrinker in $\mathbb{R}^{n+1}$. We obtain

Theorem 1.2. Let $\Sigma^{n}$ be a complete noncompact properly immersed self-shrinker in Euclidean space $\mathbb{R}^{n+1}$ with nonnegative constant $\beta \leq \inf H^{2}$. Then, there is a positive constant $C$ such that for $r \geq 1$,

$$
\operatorname{Vol}\left(B_{r}(0) \cap \Sigma\right) \leq C r^{n-2 \beta} .
$$

The upper bounds of the volume in Theorem 1.2 are optimal because $\mathbb{R}^{n}$ and the cylinder self-shrinker $S^{k}(\sqrt{2 k}) \times \mathbb{R}^{n-k}$ have the corresponding volume growth rates. In [8], Ding and Xin proved the Euclidean volume growth of a complete noncompact properly immersed self-shrinker. Theorem 1.2 implies their result.

Further, in Section 4 we study the relation among weighted volume finiteness, polynomial volume growth and properness of a self-shrinker. In the works of EckerHuisken [9] and Colding-Minicozzi [6] the polynomial volume growth plays an important role in studying the self-shrinker. It is satisfied when the self-shrinker is a time-slice of a blowup. In general, for a complete noncompact self-shrinker $\Sigma$, we show in Theorem 1.3 that the following statements are equivalent.

Theorem 1.3. For any complete immersed self-shrinker $\Sigma^{n}$ in $\mathbb{R}^{n+1}$, the following statements are equivalent:

(i) $\Sigma$ is proper;

(ii) $\Sigma$ has Euclidean volume growth; 
(iii) $\Sigma$ has polynomial volume growth;

(iv) $\Sigma$ has finite weighted volume, that is, $\int_{\Sigma} e^{-\frac{|x|^{2}}{4}} d v<\infty$.

Theorems 1.2 and 1.3 are the consequences of the corresponding general results of self-shrinkers in $\mathbb{R}^{n+p}, p \geq 1$ (see Theorems 3.1 and 4.1).

Using Theorem 1.3, we can rephrase the compactness theorem of Colding and Minicozzi [7] as the following.

Theorem 1.4 (Colding-Minicozzi [7]). Given an integer $g \geq 0$ and a constant $A>0$, the set

$$
\mathcal{S}(g, A):=\left\{\begin{array}{c}
\Sigma: \Sigma \text { is a complete smooth embedded self-shrinker in } \mathbb{R}^{3} \text { with } \\
\text { genus at most } g \text { and } \int_{\Sigma} e^{-\frac{|x|^{2}}{4}} d v \leq A
\end{array}\right\}
$$

is compact with respect to the topology of $C^{m}$ convergence on compact subsets for any $m \geq 2$.

The second application of Theorem 1.1 is about gradient shrinking solitons. Recall that a complete smooth Riemannian manifold $\left(M^{n}, g\right)$ is called a normalized gradient shrinking Ricci soliton if there is a smooth function $f$ on $M^{n}$ such that the Ricci tensor $R_{i j}$ of the metric $g$ is given by

$$
R_{i j}+\nabla_{i} \nabla_{j} f=\frac{1}{2} g_{i j}
$$

It is known that the scalar curvature $R$ of the metric $g$ is nonnegative 5 . We get

Theorem 1.5 (Theorem 5.1). Let $\left(M^{n}, g, f\right)$ be a complete noncompact gradient shrinking solition with $f$ satisfying (3) and $\beta=\inf R$. Then there exists a constant $C>0$ such that

$$
\operatorname{Vol}\left(B_{r}\left(x_{0}\right)\right) \leq C r^{n-2 \beta}
$$

for $r \geq 1$, where $C$ is a constant depending on $x_{0}$ and $\operatorname{Vol}\left(B_{r}\left(x_{0}\right)\right)$ denotes the volume of the geodesic ball of $M$ of radius $r$ centered at $x_{0} \in M$.

Since the scalar curvature $R$ is nonnegative ([5]), Theorem 1.5] implies the estimate of Euclidean volume growth which has been obtained by Cao and the second author in [4. The volume upper bounds of the geodesic ball $B_{r}\left(x_{0}\right)$ in Theorem 1.5 are sharp by the examples such as the Gaussian shrinker and cylinder shrinking solitons. After the first version was posted on the arXiv, we were informed that Theorem 1.5 had been proved by Zhang [14. In 14, the author used the method in [4] to prove the result.

It has been proved (Cao-Chen-Zhu [3]) that any 3-dimensional complete noncompact nonflat shrinking gradient soliton is necessarily the round cylinder $\mathbb{S}^{2} \times \mathbb{R}$ or one of its $\mathbb{Z}_{2}$ quotients. But for higher dimensions, one has no classification of complete noncompact gradient shrinking solitons without any assumptions on curvatures.

\section{Volume Growth estimate}

In this section, we will prove Theorem 1.1. The first part of our argument is similar to the proof of Ding and Xin in the case of self-shrinkers. We have the following. 
Theorem 2.1 (Theorem 1.1). Let $(M, g)$ be a complete noncompact Riemannain manifold. Let $f$ be a proper $C^{\infty}$ function on $M$. If $|\nabla f|^{2} \leq f$ on $D_{r}$ for all $r$ and

$$
\Delta_{f} f+f \leq k
$$

for some constant $k$, then the weighted volume $\int_{M} e^{-f} d v<+\infty$ and

$$
V(r) \leq C r^{2 k}
$$

for $r \geq 1$, where $C$ is a constant depending only on $\int_{M} e^{-f} d v$.

Proof. Since $f$ is proper, it is well defined that the integral

$$
\begin{gathered}
I(t)=\frac{1}{t^{k}} \int_{\bar{D}_{r}} e^{-\frac{f}{t}} d v, \quad t>0, \\
I^{\prime}(t)=t^{-k-1} \int_{\bar{D}_{r}}\left(-k+\frac{f}{t}\right) e^{-\frac{f}{t}} d v .
\end{gathered}
$$

On the other hand,

$$
\begin{aligned}
\int_{\bar{D}_{r}} \operatorname{div}\left(e^{-\frac{f}{t}} \nabla f\right) d v & =\int_{\bar{D}_{r}} e^{-\frac{f}{t}}\left(\Delta f-\frac{1}{t}|\nabla f|^{2}\right) d v \\
& \leq \int_{\bar{D}_{r}} e^{-\frac{f}{t}}\left(|\nabla f|^{2}-f+k-\frac{1}{t}|\nabla f|^{2}\right) d v \\
& =\int_{\bar{D}_{r}} e^{-\frac{f}{t}}\left(\frac{t-1}{t}|\nabla f|^{2}-f+k\right) d v \\
& \leq \int_{\bar{D}_{r}} e^{-\frac{f}{t}}\left(\frac{t-1}{t} f-f+k\right) d v, \quad t \geq 1 \\
& =\int_{\bar{D}_{r}} e^{-\frac{f}{t}}\left(-\frac{1}{t} f+k\right) d v .
\end{aligned}
$$

Substituting (77) into (6) gives

$$
I^{\prime}(t) \leq-t^{-k-1} \int_{\bar{D}_{r}} \operatorname{div}\left(e^{-\frac{f}{t}} \nabla f\right) d v .
$$

At the regular value $r$ of $f$ and for $t \geq 1$, by Stokes' Theorem, we have

$$
\begin{aligned}
I^{\prime}(t) & \leq-t^{-k-1} \int_{\partial D_{r}}\left\langle e^{-\frac{f}{t}} \nabla f, \frac{\nabla f}{|\nabla f|}\right\rangle d v \\
& =-t^{-k-1} \int_{\partial D_{r}} e^{-\frac{f}{t}}|\nabla f| d v \leq 0 .
\end{aligned}
$$

Integrating $I^{\prime}(t)$ over $t$ from 1 to $r^{2}>1$, we get

$$
r^{-2 k} \int_{\bar{D}_{r}} e^{-\frac{f}{r^{2}}} d v \leq \int_{\bar{D}_{r}} e^{-f} d v
$$

Since the integrals in (8) are upper semi-continuous for all $r$, (8) holds for all $r \geq 1$.

Note that $2 \sqrt{f} \leq r$ over $\bar{D}_{r}$. (8) implies that for all $r \geq 1$,

$$
\begin{aligned}
e^{-\frac{1}{4}} r^{-2 k} \int_{\bar{D}_{r}} d v & \leq r^{-2 k} \int_{\bar{D}_{r}} e^{-\frac{f}{r^{2}}} d v \\
& \leq \int_{\bar{D}_{r}} e^{-f} d v
\end{aligned}
$$


Note that

$$
\begin{aligned}
\int_{\bar{D}_{r}} e^{-f} d v-\int_{\bar{D}_{r-1}} e^{-f} d v & =\int_{\bar{D}_{r} \backslash \bar{D}_{r-1}} e^{-f} d v \\
& \leq e^{-\frac{(r-1)^{2}}{4}} \int_{\bar{D}_{r} \backslash \bar{D}_{r-1}} d v \\
& \leq e^{-\frac{(r-1)^{2}}{4}} \int_{\bar{D}_{r}} d v
\end{aligned}
$$

By (9) and (10), we have

$$
\int_{\bar{D}_{r}} e^{-f} d v-\int_{\bar{D}_{r-1}} e^{-f} d v \leq e^{\frac{1}{4}} r^{2 k} e^{-\frac{(r-1)^{2}}{4}} \int_{\bar{D}_{r}} e^{-f} d v
$$

We can choose a constant $r_{0}$ such that $e^{\frac{1}{4}} r^{2 k} e^{-\frac{(r-1)^{2}}{4}}<e^{-r}$, for $r>r_{0}$. Then (11) implies that

$$
\int_{\bar{D}_{r}} e^{-f} d v \leq \frac{1}{1-e^{-r}} \int_{\bar{D}_{r-1}} e^{-f} d v
$$

Then for any positive integer $N$, we have

$$
\int_{\bar{D}_{r+N}} e^{-f} d v \leq\left(\prod_{i=0}^{N} \frac{1}{1-e^{-(r+i)}}\right) \int_{\bar{D}_{r-1}} e^{-f} d v<\infty .
$$

This implies that $\int_{M} e^{-f} d v<+\infty$. Moreover by (9),

$$
e^{-\frac{1}{4}} r^{-2 k} \int_{\bar{D}_{r}} d v \leq \int_{\bar{D}_{r}} e^{-f} d v \leq \int_{M} e^{-f} d v
$$

So for all $r \geq 1, V(r) \leq C r^{2 k}$.

\section{Application to SELF-Shrinkers}

In this section, we discuss the volume of self-shrinkers of mean curvature flow by using Theorem 2.1. Let $\Sigma$ be an $n$-dimensional submanifold isometrically immersed in $\mathbb{R}^{n+p}$. Let $A(V, W)=\bar{\nabla}_{V} W-\nabla_{V} W, V, W \in T \Sigma$ denote the second fundamental form and $\mathbf{H}$ denote the mean curvature vector of $\Sigma$ given by $\mathbf{H}=\sum_{i=1}^{n} A\left(e_{i}, e_{i}\right)$, where $\left\{e_{i}\right\}$ is a local orthonormal frame field of $\Sigma . \Sigma$ is said to be a self-shrinker in $\mathbb{R}^{n+p}$ if it satisfies

$$
\mathbf{H}=-\frac{x^{N}}{2},
$$

where $x$ denotes the position vector and $(\cdot)^{N}$ denotes the orthogonal projection into the normal bundle $N \Sigma$ of $\Sigma$.

Let $f(x)=\frac{|x|^{2}}{4}$. Then $\bar{\nabla} f=\frac{x}{2},|\bar{\nabla} f|^{2}=\frac{|x|^{2}}{4}$,

$$
\begin{aligned}
f-|\nabla f|^{2} & =f-|\bar{\nabla} f|^{2}+\left|(\bar{\nabla} f)^{N}\right|^{2}=\frac{\left|x^{N}\right|^{2}}{4}=|\mathbf{H}|^{2} \geq 0, \\
\Delta_{\Sigma} f & =\frac{1}{4} \sum_{i=1}^{n} \bar{D}^{2}\left(|x|^{2}\right)\left(e_{i}, e_{i}\right)+\frac{1}{4}\left\langle\mathbf{H}, \bar{\nabla}|x|^{2}\right\rangle \\
& =\frac{n}{2}-\frac{1}{4}\left\langle x^{N}, x\right\rangle^{2} \\
& =\frac{n}{2}-f+|\nabla f|^{2} .
\end{aligned}
$$


Denote by $B_{r}(0)=\left\{x \in \mathbb{R}^{n+1} ;|x|<r\right\}$ the ball in $\mathbb{R}^{n+1}$ of radius $r$ centered at the origin.

Theorem 3.1. Let $\Sigma^{n}$ be a complete noncompact properly immersed self-shrinker in Euclidean space $\mathbb{R}^{n+p}$ with nonnegative constant $\beta \leq \inf |\mathbf{H}|^{2}$. Then, there is a positive constant $C$ such that for $r \geq 1$,

$$
\operatorname{Vol}\left(B_{r}(0) \cap \Sigma\right) \leq C r^{n-2 \beta} .
$$

Proof. By (15), inf $\left\{f-|\nabla f|^{2}\right\}=\inf |\mathbf{H}|^{2} \geq \beta \geq 0$. Take $\bar{f}=f-\beta$. Then

$$
\begin{gathered}
\bar{f}-|\bar{\nabla} f|^{2}=f-|\nabla f|^{2}-\beta \geq 0, \\
\Delta_{\Sigma} \bar{f}=\frac{n}{2}-\beta-\bar{f}+|\nabla \bar{f}|^{2} .
\end{gathered}
$$

The properness of the immersion of $\Sigma$ is the properness of $f$ on $\Sigma$, and hence $\bar{f}$ is proper. The above implies that $\bar{f}$ satisfies the assumption of Theorem 2.1] Denote

$$
D_{r}=\{x \in \Sigma: 2 \sqrt{\bar{f}}<r\} .
$$

Then

$$
D_{r}=\left\{x \in \Sigma:|x|<\sqrt{r^{2}+4 \beta}\right\} .
$$

By Theorem 2.1

$$
\operatorname{Vol}\left(D_{r}\right) \leq C r^{n-2 \beta}
$$

that is,

$$
\operatorname{Vol}\left(B_{\sqrt{r^{2}+4 \beta}}(0) \cap \Sigma\right) \leq C r^{n-2 \beta} .
$$

So

$$
\operatorname{Vol}\left(B_{r}(0) \cap \Sigma\right) \leq C r^{n-2 \beta}
$$

In particular, let $\Sigma^{n}$ be a self-shrinker in $\mathbb{R}^{n+1}$. Since $\mathbf{H}=-H \mathbf{n}$, where $H=-\left\langle\nabla_{e_{i}} e_{i}, \mathbf{n}\right\rangle$ and $\mathbf{n}$ denote the mean curvature and the outward unit normal respectively, $\Sigma$ satisfies

$$
H=\frac{\langle x, \mathbf{n}\rangle}{2} .
$$

So we have Theorem 1.2

Remark 3.1. Take the cylinder self-shrinker $S^{k}(\sqrt{2 k}) \times \mathbb{R}^{n-k}, 0 \leq k \leq n, H=\frac{\sqrt{k}}{\sqrt{2}}$, $n-2 \beta=n-k$. Theorem 3.1 implies that $\operatorname{Vol}\left(B_{r}(0) \cap \Sigma\right) \leq C r^{n-k}$, which is sharp. The constant $C$ in the theorem depends only on the integral $\int_{\Sigma} e^{-\frac{|x|^{2}}{4}} d v<\infty$ and $\beta$ explicitly as seen in the proof. Take $\beta=0$ in Theorem 3.1. We have the Euclidean growth upper bound proved by Ding and Xin [8]. 


\section{Properness, polynomial volume growth and FINITE WEIGHTED VOLUME FOR SELF-SHRINKERS}

In this section, we discuss the equivalence of properness, polynomial volume growth and finite weighted volume for complete self-shrinkers. If $\Sigma$ is an $n$-dimensional submanifold in $\mathbb{R}^{n+p}, p \geq 1, \Sigma$ is said to have polynomial volume growth if there exist constants $C$ and $d$ so that for all $r \geq 1$,

$$
\operatorname{Vol}\left(B_{r}(0) \cap \Sigma\right) \leq C r^{d} .
$$

When $d=n$ in (18), $\Sigma$ is said to have Euclidean volume growth. We get the following.

Theorem 4.1. For any complete $n$-dimensional immersed self-shrinker $\Sigma^{n}$ in $\mathbb{R}^{n+p}, p \geq 1$, the following statements are equivalent:

(i) $\Sigma$ is proper;

(ii) $\Sigma$ has Euclidean volume growth;

(iii) $\Sigma$ has polynomial volume growth;

(iv) $\Sigma$ has finite weighted volume, that is, $\int_{\Sigma} e^{-\frac{|x|^{2}}{4}} d v<\infty$.

Proof. $(i) \Rightarrow($ ii $)$ has been proved in Theorem 3.1. (ii) $\Rightarrow($ iii $)$ and $(i i i) \Rightarrow(i v)$ are obvious. So we only need to prove that $(i v)$ implies $(i)$. On the contrary, if $\Sigma$ is not properly immersed, there exists a number $R>0$ such that the pre-image $E \subseteq \Sigma$ of $\bar{B}_{R}$ is not compact in $\Sigma$. Then for a positive constant $a>0$, there exists a sequence $\left\{p_{k}\right\}$ of points in $E$ with $d_{\Sigma}\left(p_{k}, p_{j}\right) \geq a>0$ for any $k \neq j$, where $d_{\Sigma}$ denotes the intrinsic distance in $\Sigma$. Denote by $B_{r}^{\Sigma}(p)$ the geodesic ball of $\Sigma$ of radius $r$ centered at $p \in \Sigma$. So $B_{\frac{a}{2}}^{\Sigma}\left(p_{k}\right) \cap B_{\frac{a}{2}}^{\Sigma}\left(p_{j}\right)=\emptyset$ for any $k \neq j$. Choose $a<2 R$. Then $B_{\frac{a}{2}}^{\Sigma}\left(p_{k}\right) \subset B_{2 R}$. Since

$$
\mathbf{H}=-\frac{x^{N}}{2},
$$

for any $p \in \Sigma \cap B_{2 R}$,

$$
|\mathbf{H}|(p) \leq \frac{|x|}{2} \leq \frac{2 R}{2}=R .
$$

We claim that there is a constant $C$ depending only on $R$ such that $\operatorname{vol}\left(B_{\frac{a}{2}}^{\Sigma}\left(p_{k}\right)\right) \geq$ $C a^{n}$ when $0<a \leq \min \left\{2 R, \frac{n}{2 R}\right\}$ is suitably small.

In fact, for $\left.p \in B_{\frac{a}{2}}^{\Sigma}\left(p_{k}\right)\right)$, the extrinsic distance function $r_{k}(p):=\mathrm{d}\left(p, p_{k}\right)$ from $p_{k}$ satisfies

$$
\begin{aligned}
\Delta_{\Sigma} r_{k}^{2} & =2 n+2\left\langle\mathbf{H}, r_{k} \bar{\nabla} r_{k}\right\rangle \\
& \geq 2 n-2|\mathbf{H}| r_{k} \\
& \geq 2 n-2 r_{k} R .
\end{aligned}
$$

By $a \leq \frac{n}{2 R}$, we have for $0<\mu \leq \frac{a}{2}$,

$$
\begin{aligned}
\int_{B_{\mu}^{\Sigma}\left(p_{k}\right)}\left(2 n-2 R r_{k}\right) d v & \leq \int_{B_{\mu}^{\Sigma}\left(p_{k}\right)} \Delta_{\Sigma} r_{k}^{2} d v \\
& =\int_{\partial B_{\mu}^{\Sigma}\left(p_{k}\right)}\left\langle\nabla^{\Sigma} r_{k}^{2}, \nu\right\rangle d v \\
& \leq 2 \mu A(\mu),
\end{aligned}
$$


where $\nu$ denotes the outward unit normal vector of $\partial B_{\mu}^{\Sigma}\left(p_{k}\right)$ and $A(\mu)$ denotes the area of $\partial B_{\mu}^{\Sigma}\left(p_{k}\right)$. Then using the co-area formula in (22), we get

$$
\int_{0}^{\mu}(n-R s) A(s) d s \leq \int_{0}^{\mu} \int_{d_{\Sigma}\left(p, p_{k}\right)=s}\left(n-R r_{k}\right) d \sigma \leq \mu A(\mu) .
$$

This implies that

$$
(n-R \mu) V(\mu) \leq \mu V^{\prime}(\mu),
$$

where $V(\mu)$ denotes the volume of $B_{\mu}^{\Sigma}\left(p_{k}\right)$. So

$$
\frac{V^{\prime}(\mu)}{V(\mu)} \geq \frac{n}{\mu}-R
$$

Integrating (24) from $\epsilon>0$ to $\mu$, we have

$$
\frac{V(\mu)}{V(\epsilon)} \geq\left(\frac{\mu}{\epsilon}\right)^{n} e^{-R(\mu-\epsilon)}
$$

Since $\lim _{s \rightarrow 0^{+}} \frac{V(s)}{s^{n}}=\omega_{n}$,

$$
V(\mu) \geq \omega_{n} \mu^{n} e^{-R \mu} .
$$

The claim follows from (25). Now we have

$$
\begin{aligned}
\int_{\Sigma} e^{-\frac{|x|^{2}}{4}} d v & \geq \sum_{k=1}^{\infty} \int_{\left.B_{\frac{a}{2}}^{\Sigma}\left(p_{k}\right)\right)} e^{-\frac{|x|^{2}}{4}} d v \\
& \geq e^{-R^{2}} \sum_{k=1}^{\infty} \int_{B_{\frac{a}{2}}^{\Sigma}\left(p_{k}\right)} d v=+\infty
\end{aligned}
$$

This is a contradiction to the hypothesis of the finiteness of the weighted volume.

Taking $p=1$ in Theorem 4.1, we get Theorem 1.3 .

Remark 4.1. It was asked by Huai-Dong Cao if a complete self-shrinker has polynomial volume growth. If there is any example of a nonproperly immersed selfshrinker, it must have infinite weighted volume. We do not know any such examples.

\section{Application to GRAdient Shrinking SOlitons}

In this section, we consider gradient shrinking solitons of Ricci flows. A complete $n$-dimensional smooth Riemannian manifold $(M, g)$ is called a gradient shrinking Ricci soliton if there exists a smooth function $f$ on $M^{n}$ such that the Ricci tensor $R_{i j}$ of the metric $g$ is given by

$$
R_{i j}+\nabla_{i} \nabla_{j} f=\rho g_{i j}
$$

for some positive constant $\rho>0$. By scaling $g_{i j}$ one can normalize $\rho=\frac{1}{2}$ so that

$$
R_{i j}+\nabla_{i} \nabla_{j} f=\frac{1}{2} g_{i j} .
$$

Taking the trace in (27), we have

$$
R+\Delta f=\frac{n}{2} .
$$


It was proved by Hamilton [10 that there is a constant $C_{0}$ such that the scalar curvature $R$ and $f$ satisfy

$$
R+|\nabla f|^{2}-f=C_{0} .
$$

Since B.L. Chen [5] proved that a complete gradient shrinking soliton has nonnegative scalar curvature (cf. Proposition 5.5 [1]), we can choose a new $f$, still denoted by $f$, such that $C_{0}$ is equal to the infimum of $R$. Denote by $\beta=\inf R$. Hence for this $f, 0 \leq|\nabla f|^{2} \leq f$ and thus $(M, g)$ is a gradient shrinking Ricci soliton with nonnegative potential function $f$ and

$$
\Delta f-|\nabla f|^{2}+f=\frac{n}{2}-\beta .
$$

Using Theorem 2.1, we get the following.

Lemma 5.1. Let $\left(M^{n}, g, f\right)$ be a complete noncompact gradient shrinking solition with $f$ satisfying (27) and (29) with $\beta=\inf R$. Then $\int_{M} e^{-f} d v<\infty$ and

$$
V(r) \leq C r^{n-2 \beta}
$$

for $r \geq 1$, where $C$ is a constant depending only on $\int_{M} e^{-f} d v$.

Proof. It suffices to show the properness of $f$. Take $\bar{f}=f+\beta$. In 4 , it is proved that the potential function $\bar{f}$ satisfies for $r$ sufficiently large

$$
\frac{1}{4}\left(r(x)-c_{1}\right)^{2} \leq \bar{f}(x) \leq \frac{1}{4}\left(r(x)+c_{2}\right)^{2},
$$

where $r(x)=d\left(x_{0}, x\right)$ is the distance function from some fixed point $x_{0} \in M, c_{1}$ and $c_{2}$ are positive constants depending only on $n$ and the geometry of $g_{i j}$ on the unit ball $B_{x_{0}}(1)$. Hence $\bar{f}$ is proper and thus $f$ is also.

Theorem 5.1 (Theorem 1.5). Let $\left(M^{n}, g, f\right)$ be a complete noncompact gradient shrinking solition with $f$ satisfying (27) and $\beta=\inf R$. Then there exists a constant $C>0$ such that

$$
\operatorname{Vol}\left(B_{r}\left(x_{0}\right)\right) \leq C r^{n-2 \beta}
$$

for $r \geq 1$, where $C$ is a constant depending on $x_{0}$ and $\operatorname{Vol}\left(B_{r}\left(x_{0}\right)\right)$ denotes the volume of the geodesic ball of $M$ of radius $r$ centered at $x_{0} \in M$.

Proof. Take $\tilde{f}=f+\beta-C_{0}$. Then $\tilde{f}$ satisfies Lemma 5.1. So let $D_{r}=\{x \in M$ : $2 \sqrt{\tilde{f}}<r\}$. Then

$$
V\left(D_{r}\right) \leq C r^{n-2 \beta}
$$

for $r \geq 1$. By (30), it is easy to obtain $B_{r}\left(x_{0}\right) \subseteq D_{r+c}$ for some constant $c$ and sufficiently large $r$.

Remark 5.1. The volume upper bounds of the level set $D_{r}$ and the geodesic ball $B_{r}\left(x_{0}\right)$ in Theorem 5.1 are optimal: for example, the Gaussian shrinker, namely the flat Euclidean space $\left(\mathbb{R}^{n}, g_{0}\right)$ with the potential function $f=|x|^{2} / 4$ and cylinder shrinking solitons $\left(S^{n-k} \times \mathbb{R}^{k}, g\right), k \geq 1$, where $g=2(n-k-1) g_{S^{n-k}}+g_{\mathbb{R}^{k}}$ and $f(\theta, x)=\frac{|x|^{2}}{4}, \theta \in S^{n-k}, x \in \mathbb{R}^{k}$.

Remark 5.2. Since $\beta=\inf R \geq 0$, Theorem 5.1 implies the Euclidean volume growth estimate of Cao and the second author [4. The method of the proof presented in Lemma 5.1 is different. 
Remark 5.3. By [4, the equation of the shrinking Ricci soliton gives the estimate of the potential function; we can use the finiteness of the weighted volume to conclude that the fundamental group $\pi_{1}(\Sigma)$ of $\Sigma$ is finite, which was proved by W. Wylie [13. But for a self-shrinker, the potential function comes from the restriction of the metric of the ambient space. We cannot use it to study the fundamental group of the self-shrinker.

\section{REFERENCES}

[1] Huai-Dong Cao, Geometry of complete gradient shrinking Ricci solitons, Geometry and analysis. No. 1, Adv. Lect. Math. (ALM), vol. 17, Int. Press, Somerville, MA, 2011, pp. 227-246. MR.2882424

[2] _ Recent progress on Ricci solitons, Recent advances in geometric analysis, Adv. Lect. Math. (ALM), vol. 11, Int. Press, Somerville, MA, 2010, pp. 1-38. MR2648937|(2011d:53061)

[3] Huai-Dong Cao, Bing-Long Chen, and Xi-Ping Zhu, Recent developments on Hamilton's Ricci flow, Surveys in differential geometry. Vol. XII. Geometric flows, Surv. Differ. Geom., vol. 12, Int. Press, Somerville, MA, 2008, pp. 47-112. MR2488948(2010c:53096)

[4] Huai-Dong Cao and Detang Zhou, On complete gradient shrinking Ricci solitons, J. Differential Geom. 85 (2010), no. 2, 175-185. MR2732975

[5] Bing-Long Chen, Strong uniqueness of the Ricci flow, J. Differential Geom. 82 (2009), no. 2, 363-382. MR2520796 (2010h:53095)

[6] Tobias H. Colding and William P. Minicozzi II, Generic mean curvature flow I: generic singularities, Ann. of Math. (2) 175 (2012), no. 2, 755-833.

[7] _ Smooth compactness of self-shrinkers, Comment. Math. Helv. 87 (2012), no. 1, 463475 .

[8] Qi Ding and Yuanlong Xin, Volume growth, eigenvalue and compactness for self-shrinkers. arXiv:1101.1411.

[9] Klaus Ecker and Gerhard Huisken, Mean curvature evolution of entire graphs, Ann. of Math. (2) 130 (1989), no. 3, 453-471, DOI 10.2307/1971452. MR:1025164 (91c:53006)

[10] Richard S. Hamilton, The formation of singularities in the Ricci flow, Surveys in Differential Geometry, vol. II (Cambridge, MA, 1993), Int. Press, Cambridge, MA, 1995, pp. 7-136. MR 1375255 (97e:53075)

[11] Gerhard Huisken, Asymptotic behavior for singularities of the mean curvature flow, J. Differential Geom. 31 (1990), no. 1, 285-299. MR1030675 (90m:53016)

[12] G. Perelman, The entropy formula for the Ricci flow and its geometric applications. arXiv: math.DG/0211159.

[13] William Wylie, Complete shrinking Ricci solitons have finite fundamental group, Proc. Amer. Math. Soc. 136 (2008), no. 5, 1803-1806. MR2373611(2009a:53059)

[14] Shi Jin Zhang, On a sharp volume estimate for gradient Ricci solitons with scalar curvature bounded below, Acta Math. Sin. (Engl. Ser.) 27 (2011), no. 5, 871-882. MR2786449 (2012g:53081)

Instituto de Matematica, Universidade Federal Fluminense, Niterói, RJ 24020, Brazil

E-mail address: xcheng@impa.br

Instituto de Matematica, Universidade Federal Fluminense, Niterói, RJ 24020, Brazil

E-mail address: zhou@impa.br 